\title{
Coca-Cola's secret influence on medical and science journalists
}

Clarification - This investigation (BMJ 2017;357:j1638, doi:10. 1136/bmj.j1638) was based on documents, originally obtained by the organisation US. Right to Know, and later verified for authenticity by the General Counsel's office at the University of Colorado. 